\title{
Robustness of digitally modulated signal features against variation in HF noise model
}

\author{
Alharbi Hazza ${ }^{1 *}$, Mobien Shoaib², Alshebeili Saleh ${ }^{1,2}$ and Alturki Fahd ${ }^{1}$
}

\begin{abstract}
High frequency (HF) band has both military and civilian uses. It can be used either as a primary or backup communication link. Automatic modulation classification (AMC) is of an utmost importance in this band for the purpose of communications monitoring; e.g., signal intelligence and spectrum management. A widely used method for AMC is based on pattern recognition (PR). Such a method has two main steps: feature extraction and classification. The first step is generally performed in the presence of channel noise. Recent studies show that HF noise could be modeled by Gaussian or bi-kappa distributions, depending on day-time. Therefore, it is anticipated that change in noise model will have impact on features extraction stage. In this article, we investigate the robustness of well known digitally modulated signal features against variation in HF noise. Specifically, we consider temporal time domain (TTD) features, higher order cumulants (HOC), and wavelet based features. In addition, we propose new features extracted from the constellation diagram and evaluate their robustness against the change in noise model. This study is targeting 2PSK, 4PSK, 8PSK, 16QAM, 32QAM, and 64QAM modulations, as they are commonly used in HF communications.
\end{abstract}

Keywords: Digital modulation features, temporal time domain features, higher order cumulants, wavelet decomposition, constellation diagram, bi-kappa noise, HF band

\section{Introduction}

Automatic modulation classification (AMC) is the process of identifying modulation type of a detected signal without prior information. This technique has both military and civilian applications, and is currently an important research subject in the design of cognitive radios [1-3]. AMC is a complex task especially in a non co-operative environment as in high frequency (HF) communications, where transmission is affected by atmospheric conditions and other transmission interferences [4].

AMC methods are grouped into two categories: likelihood based (LB) and feature based (FB) methods. LB methods have two steps: calculating the likelihood function of the received signal for all candidate modulations, and then using maximum likelihood ratio test (MLRT) for decision-making. In FB methods, features are first extracted from the received signal and then applied to a

\footnotetext{
* Correspondence: hazza.ksa@gmail.com

'Electrical Engineering Department, College of Engineering, King Saud University, Riyadh, Saudi Arabia

Full list of author information is available at the end of the article
}

classifier in order to recognize the modulation type. Most of the recent literatures use the FB methods due to their low processing complexity and high performance [5]. For more details about AMC methods with a comprehensive literature review, the reader is referred to [6].

Figure 1 shows the classification task in a smart radio. The task of the signal detection block is to identify signal transmission, while the AMC contains a feature extractor followed by a classifier. The classifier can be based on fixed threshold as in decision tree methods, or based on pattern recognition (PR) methods as in artificial neural networks (ANNs) and support vector machines (SVM) $[7,8]$. Most of the features used in literature are based on wavelet $[9,10]$, temporal time domain (TTD) analysis [11-13], and higher order cumulants (HOC) [14-16]. These features are generally extracted under the assumption that the modulated signals are corrupted by additive white Gaussian noise (AWGN). Although this assumption is valid in many communication environments, recent studies show that HF noise changes between AWG and bi-kappa

\section{SpringerOpen ${ }^{\odot}$}

(C) 2011 Hazza et al; licensee Springer. This is an Open Access article distributed under the terms of the Creative Commons Attribution License (http://creativecommons.org/licenses/by/2.0), which permits unrestricted use, distribution, and reproduction in any medium, provided the original work is properly cited. 


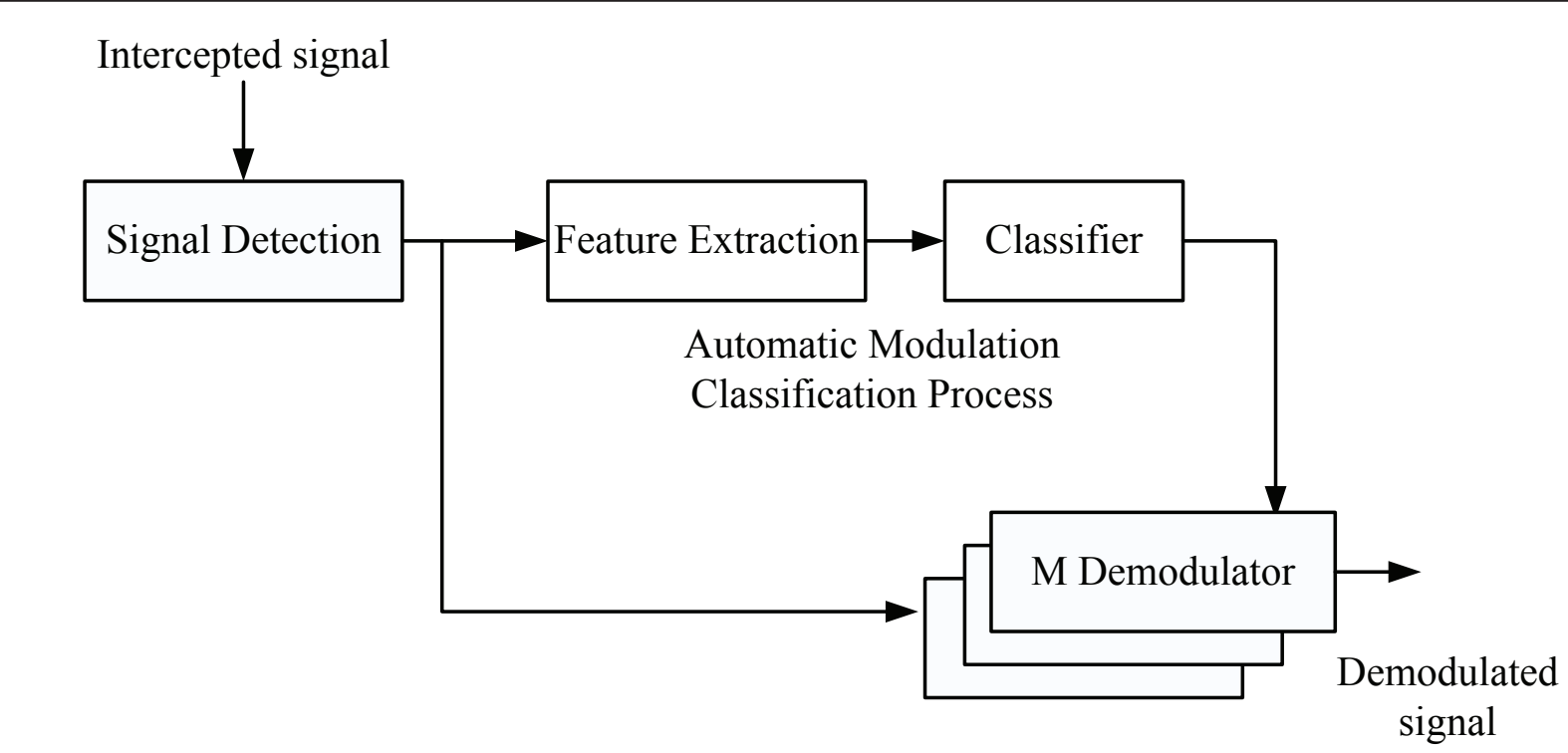

Figure 1 AMC based receiver architecture using feature based methods.

distributions $[17,18]$. The effect of these two noise distributions has been taken into account during the design of the AMC algorithms proposed in [19]. The work shows that the change in noise model affects the classification performance, especially at low signal-tonoise ratio (SNR). Therefore, the robustness of commonly used features against variation in noise models needs to be investigated so that more reliable AMC algorithms can be designed for HF signals.

In this paper, we first examine the effect of Gaussian and bi-kappa noise models on wavelet, HOC, and TTD features, when these features are considered for the classification of single carrier modulations commonly used in HF band: 2PSK, 4PSK, 8PSK, 16QAM, 32QAM, and 64QAM [20]. Second, we propose new features based on maximum dissimilarity measures (MDM) in constellation diagram and evaluate their robustness against the change in noise model. Note that the contribution of this article is pertaining to the features extraction stage; hence the results obtained are independent of the classifier being used. However, these results will greatly serve the classifier design stage, as this stage can be based on features that are robust with respect to noise models.

The organization of the article is as follows. 'Signal model' and 'Noise model' sections present signal and channel noise models, respectively. 'Commonly used signal features' section introduces the TTD, HOC, and wavelet based features. 'Proposed features' section presents the proposed features. 'Simulation results' section presents results showing the robustness of the different features against the variation in noise model. 'Conclusion' section presents concluding remarks.

\section{Signal model}

The general form of received signal encompassing all modulation schemes under consideration is given by [21]:

$$
r(t)=\operatorname{Re}\left\{C(t) e^{j 2 \pi f_{c} t}\right\}+n(t)
$$

where $C(t)$ is the complex envelope of modulated signal, $n(t)$ is band limited noise, $f_{c}$ is the carrier frequency, and $\operatorname{Re}\{\}$ denotes the real part. The complex envelope is characterized by the constellation points $C_{k}$, signal power $E$, and pulse shaping function $p(t)$. For Nsymbols with periodicity $T$, the general form of complex envelope can be expressed as:

$$
C(t)=\sqrt{E} \sum_{k}^{N} C_{k} p(t-k T)
$$

For MPSK modulation, $C_{k} \in\left\{e^{-j 2 \pi m / M_{1}}\right\}$, where $m=0$, $1, \ldots, m-1$. For MQAM modulations, $C_{k} \in a_{k}+j b_{k}, m=$ $0,1, \ldots,(M)^{1 / 2} / 2$, and

\section{Noise model}

Noise model assumed in most of the research related to AMC is AWGN. This research focuses on AMC in HF band, where the AWGN assumption no longer remains valid for all transmission times $[17,18]$. Instead, the noise varies between AWGN and bi-kappa distributions. 


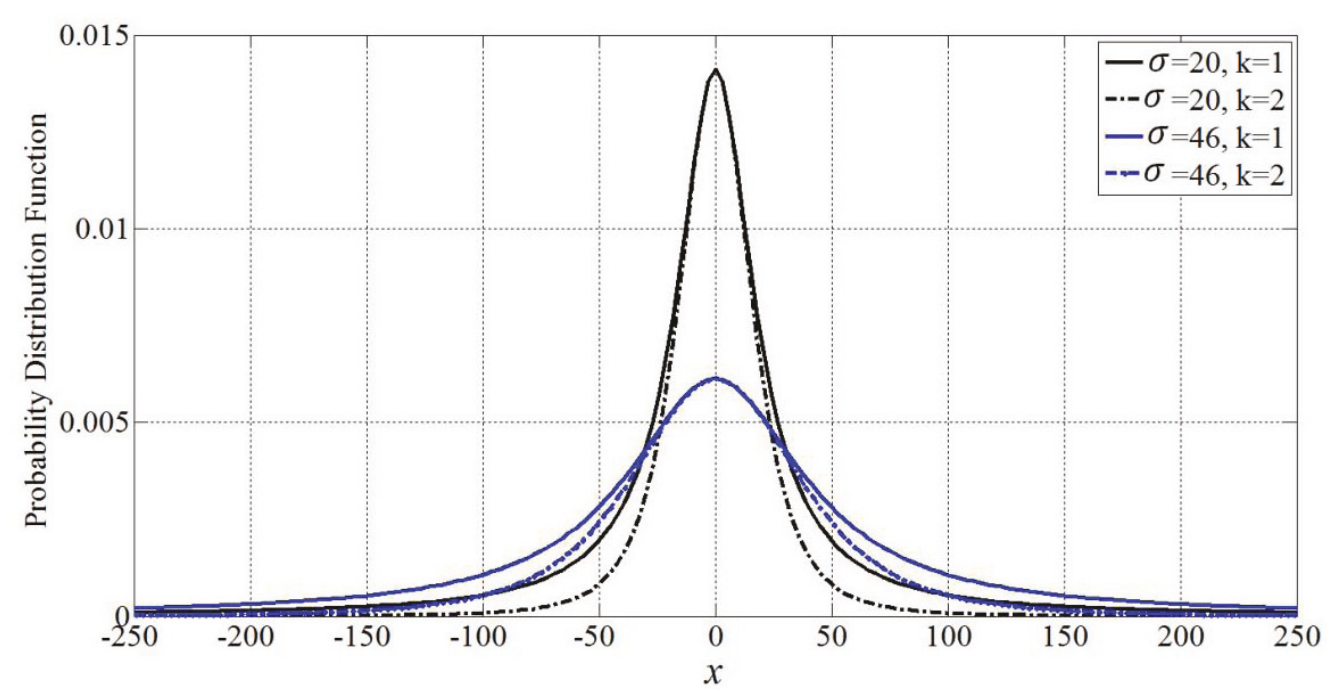

Figure 2 Probability distribution function of bi-kappa noise for different values of parameters

The bi-Kappa distribution is characterized by the following probability distribution function:

$$
p(x, k)= \begin{cases}\frac{1}{2 \sqrt{\pi} \sigma}\left[1+\frac{x^{2}}{k \sigma^{2}}\right]^{-k} & k>0 \\ \frac{1}{2 \sqrt{\pi} \sigma} & k=0 \\ \frac{1}{2 \sqrt{\pi} \sigma}\left[1-\frac{x^{2}}{k \sigma^{2}}\right]^{k} & k<0\end{cases}
$$

where $\sigma$ and $k$ are the shaping parameter and tuning factor, respectively. Practical values of these parameters are $\sigma=46, k=1.1$, and $\sigma=20, k=1$ [17]. Figure 2 shows the probability distribution function, $p(x, k)$, for different values of $\sigma$ and $k$. The figure shows that decreasing the shaping parameter produces a shaper peak and that the bi-kappa distribution approaches the AWG distribution when the tuning parameter is increased. In this work, the parameters of bi-kappa distribution are set to $\sigma=20, k=1$.

A more realistic noise model can be constructed by passing the bi-kappa noise through a band-limiting filter. The bandwidth of this filter is set to $8 \gamma_{\mathrm{s}}$ where $\gamma_{\mathrm{s}}$ is the symbol rate. This filter is practically used to minimize the transmission bandwidth. Figure 3 shows the constellation diagram of an intercepted 2PSK signal down converted to baseband for different SNR. This figure shows clearly the spiky nature of bi-kappa noise as compared to AWGN, especially at low SNR.

\section{Commonly used signal features}

This section gives the general formulas and description of commonly used signal features. Specifically, we consider the TTD, HOS, and wavelet based features.

\section{TTD features}

The variations in modulated waveforms can be described by three instantaneous values: frequency, phase, and amplitude [11,12]. All values related to these variations are defined as the TTD features. Two features will be investigated in our study. The first feature is the standard deviation of the absolute value of the centered non-linear component of the instantaneous phase defined as

$$
\sigma_{a p}=\sqrt{\frac{1}{L}\left[\sum_{a(i) t_{0} t_{\mathrm{hh}}} \varphi_{N L}^{2}(i)\right]-\left[\frac{1}{L} \sum_{a(i) t_{0} t_{\mathrm{hh}}} \mid \varphi_{N L}(i)\right]^{2}}
$$

where $\varphi_{N L}$ is the centered non-linear component of the instantaneous phase, $t_{\mathrm{th}}$ is the threshold value of the non weak signal, $L$ is the number of samples in $\varphi_{N L}$.

The second feature is the standard deviation of the absolute value of the normalized-centered instantaneous amplitude; that is,

$$
\sigma_{a a}=\sqrt{\frac{1}{N_{s}}\left[\sum_{i=1}^{N_{s}} a_{c n}^{2}(i)\right]-\left[\sum_{i=1}^{N_{s}}\left|a_{c n}(i)\right|\right]^{2}}
$$

where $N_{s}$ is the number of samples, $a_{c n}=a / m_{a}-1, a$ is the absolute value of the analytic form of the received signal, and $m_{a}$ is its sample mean value.

\section{HOC features}

The HOC are used to extract hidden information from non-Gaussian signals. In presence of AWGN, all the HOC are zero for orders greater than two. This makes these features attractive to classify modulated signals 


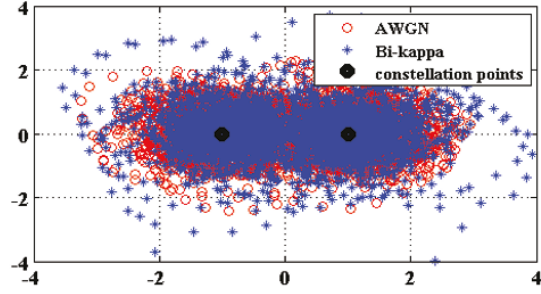

(a) Intercepted signal at $\mathrm{SNR}=0 \mathrm{~dB}$

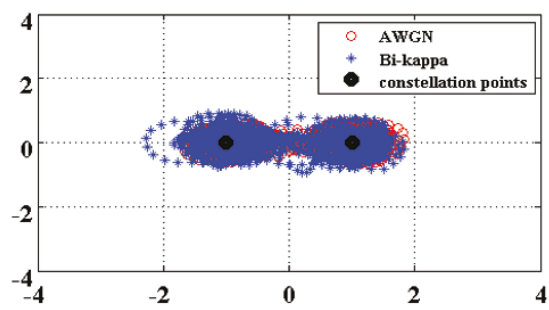

(c) Intercepted signal at $\mathrm{SNR}=10 \mathrm{~dB}$

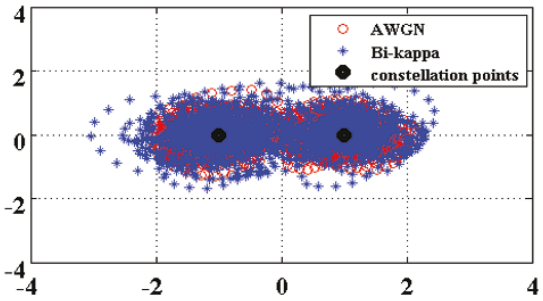

(b) Intercepted signal at $\mathrm{SNR}=5 \mathrm{~dB}$

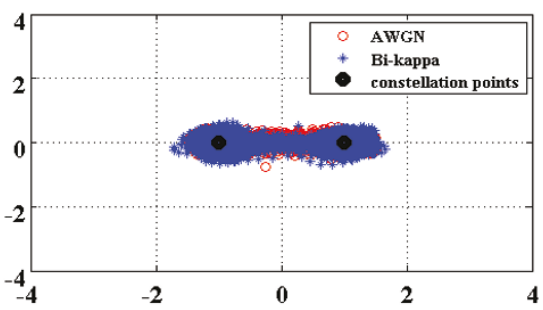

(d) Intercepted signal at $\mathrm{SNR}=15 \mathrm{~dB}$

Figure 3 Effect of HF noise models on the intercepted signals

corrupted by AWGN. Fourth and sixth HOC considered in this study are defined as follows [14-16]:

$$
\begin{aligned}
& C_{42}=M_{42}-\left|M_{20}\right|^{2}-2 M_{21}{ }^{2} \\
& C_{63}=M_{63}-9 C_{42} C_{21}-6 C_{21}{ }^{3}
\end{aligned}
$$

where $C_{21}$ is the average power and $M_{p q}$ is the joint moment. The later can be calculated for any values of $p$ and $q$ using the following equation:

$$
M_{p q}=E\left\{x^{p-q}(x *)^{q}\right\}
$$

where $x^{*}$ denotes complex conjugate and $\mathrm{E}\{\}$ is the expectation operation. Table 1 shows the theoretical cumulants for the considered modulation schemes.

\section{Wavelet features}

Wavelet transform preserves the time information while providing the frequency information of an analyzed signal. This makes it a good candidate for AMC. As shown in Figure 4, features extraction using wavelet transform passes through three steps: wavelet decomposition using Haar mother waveform, median filtering, and finally calculation of standard deviation [22]. Robustness of

Table 1 Theoretical values of HOC for digital modulations

\begin{tabular}{lll}
\hline Signal & $\boldsymbol{C}_{\mathbf{4 2}}$ & $\boldsymbol{C}_{\mathbf{6 3}}$ \\
\hline 2PSK & -2 & 13 \\
4PSK & -1 & 4 \\
8PSK & -1 & 4 \\
16QAM & -0.68 & 2.08 \\
32QAM & -0.69 & 2.11 \\
64QAM & -0.62 & 1.8 \\
\hline
\end{tabular}

wavelet features against noise model has been tested at level three and four.

\section{Proposed features}

The PSK and QAM modulations are represented by a constellation diagram in which the modulation symbols are depicted in terms of phase and amplitude variations. This diagram is extracted from the analytic form of the IF signal by multiplication with the complex conjugate of the carrier frequency. Many AMC algorithms are designed using features based on constellation diagram. These algorithms use different classification techniques that include maximum likelihood [23], genetic algorithms [24,25], modified Chi-squared test [26], and subtractive clustering [27]. In this article, we propose a different use of constellation diagram by extracting features based on maximum dissimilarity measures (MDM), firstly to distinguish between different modulation types, such as QAM and PSK signals, and secondly to find the order of a particular modulation. MDM features depend on calculating the dissimilarity between different constellation diagrams after signals normalization. That is, features are extracted from the distance (or dissimilarity measures) between the complex envelope of the received signal and set of reference constellation points for a particular modulation scheme. These reference constellation points are defined by their amplitudes and phases [21]. MDM are computed after normalizing both the received and reference constellation points to their mean values. The dissimilarity function is defined as [28]:

$$
d_{\max }(\mathrm{x}, \mathrm{p})=\max _{x \in \mathrm{x}, \mathrm{p} \in \mathrm{p}}(d(x, p))
$$




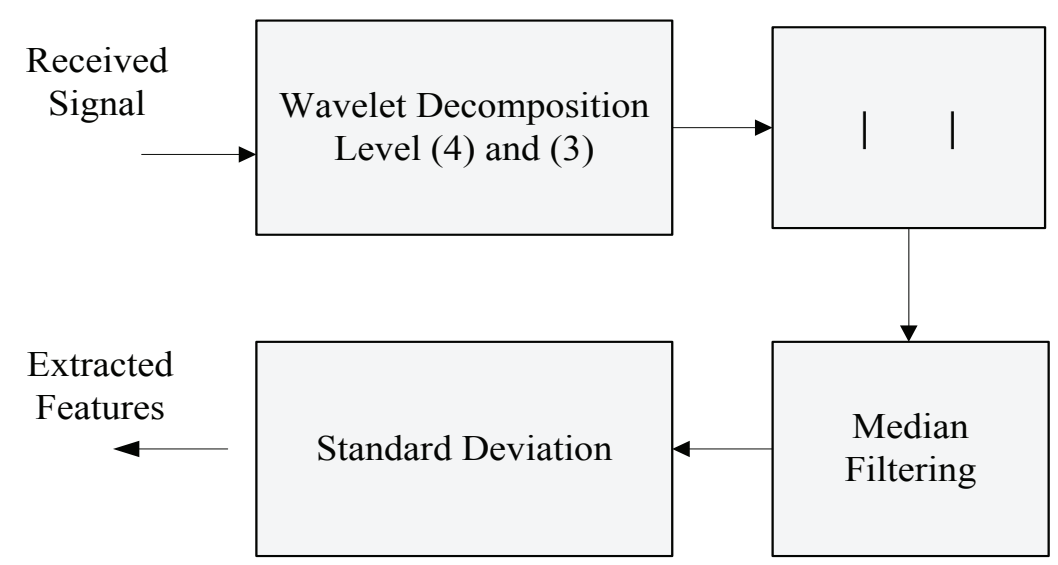

Figure 4 Steps for wavelet features extraction

where $\mathrm{d}$ is the Euclidian distance between the complex envelope of intercepted signal $\times$ and reference constellation points $p$. For feature extraction, the signal $\times$ is randomly generated at a particular SNR. A dissimilarity vector $d$, whose entries are the distance between a randomly generated constellation point of $\mathrm{x}$ and $M$ reference constellation points $\mathrm{p}$, can be obtained. The element of maximum value of vector $d$ is averaged over several independent runs, and then selected as the desired feature. In practice, the mean and/or standard deviation of $\mathrm{d}_{\max }(\mathrm{x}$, p) will have values based on the noise level.

Table 2 shows five proposed features related to the MDM, each of which is responsible for discriminating a specific modulation type or modulation order. As shown in Table 1, the first feature $d_{1}$ is used to discriminate between QAM and PSK signals, while $d_{2}$ is used to discriminate between 2PSK and other PSK signals of higher orders. For further details see Table 2.
Figures 5, 6, 7, 8, and 9 show relevant variations of proposed features as a function of SNR. The results are averaged over 100 independent realizations and displayed for AWGN. Clearly, these figures show that the proposed features have potential applications in $\mathrm{AMC}$, as they can be used in conjunction with decision tree or machine learning techniques for signal classification.

Effect of noise model on the proposed features will be discussed in the next section.

\section{Simulation results}

To evaluate the robustness of presented features, all the modulations schemes under test were generated in presence of band-limited AWGN and bi-kappa noise, where the bandwidth of the band-limiting filter is $8 \gamma_{s}$; this process is practically used to avoid high bandwidth

Table 2 Proposed features

\begin{tabular}{|c|c|c|c|}
\hline Feature & $\mathrm{p}$ & $\mathrm{x}$ & Suggested use \\
\hline$\overline{d_{1}}=\operatorname{std}\left(d_{\max }(x, p)\right)$ & 8PSK & $\begin{array}{l}\text { 2PSK } \\
\text { 4PSK } \\
\text { 8PSK } \\
\text { 16QAM } \\
\text { 32QAM } \\
\text { 64QAM }\end{array}$ & Discrimination between MPSK and MQAM \\
\hline$d_{2}=$ meand $\left._{\max }(x, p)\right)$ & 2PSK & $\begin{array}{l}\text { 2PSK } \\
4 P S K \\
8 P S K\end{array}$ & Discrimination between 2PSK and (4PSK, 8PSK) \\
\hline$d_{3}=\operatorname{mean}\left(d_{\max }(x, p)\right)$ & 4PSK & $\begin{array}{l}\text { 4PSK } \\
8 P S K\end{array}$ & Discrimination between 4PSK and 8PSK \\
\hline$d_{4}=\operatorname{mean}\left(d_{\max }(x, p)\right)$ & 16QAM & $\begin{array}{l}\text { 16QAM } \\
\text { 32QAM } \\
\text { 64QAM }\end{array}$ & Discrimination between 16QAM and (32QAM, 64QAM) \\
\hline$d_{5}=\operatorname{std}\left(d_{\max }(x, p)\right)$ & 64QAM & $\begin{array}{l}\text { 32QAM } \\
\text { 64QAM }\end{array}$ & Discrimination between 64QAM and 32MQAM \\
\hline
\end{tabular}




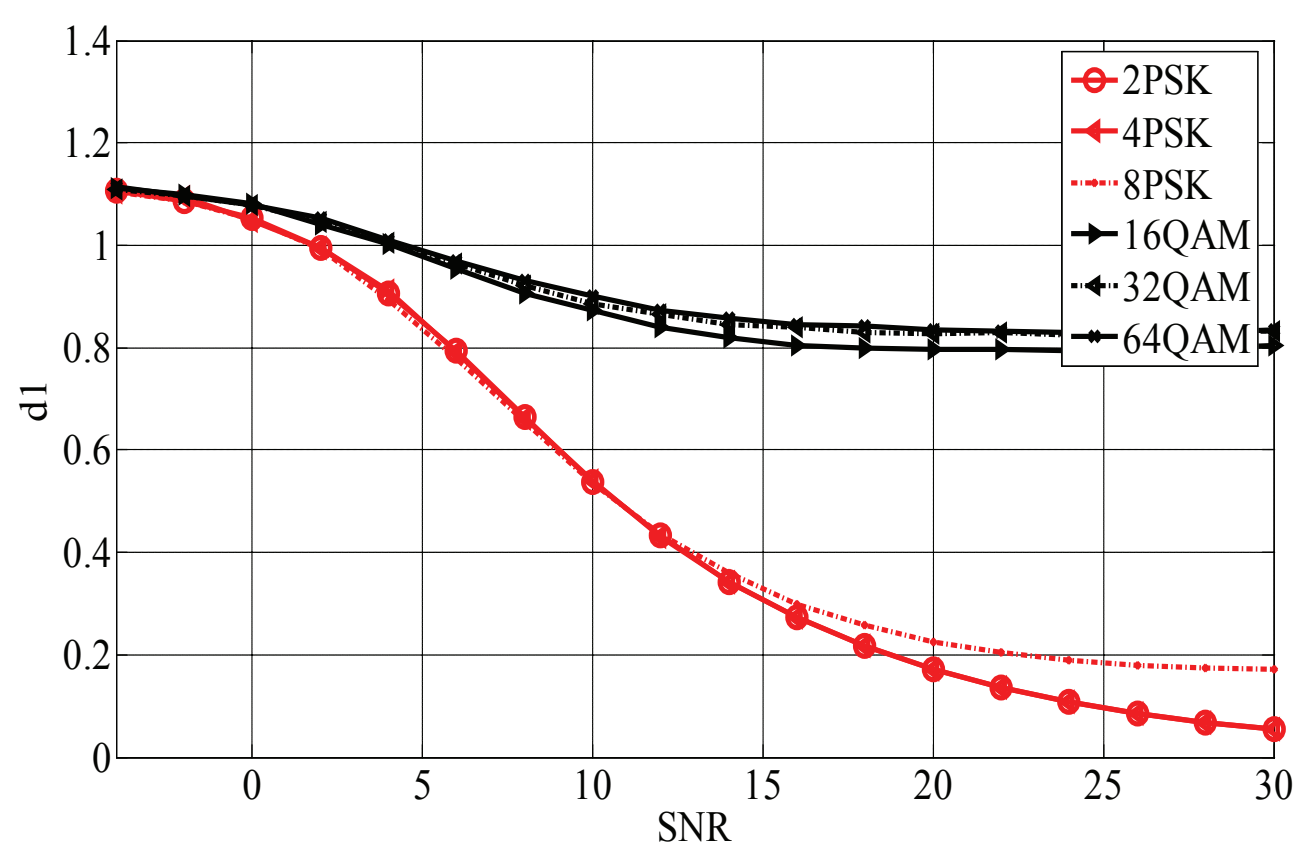

Figure $5 d_{1}$ for the discrimination between MPSK and MQAM signals.

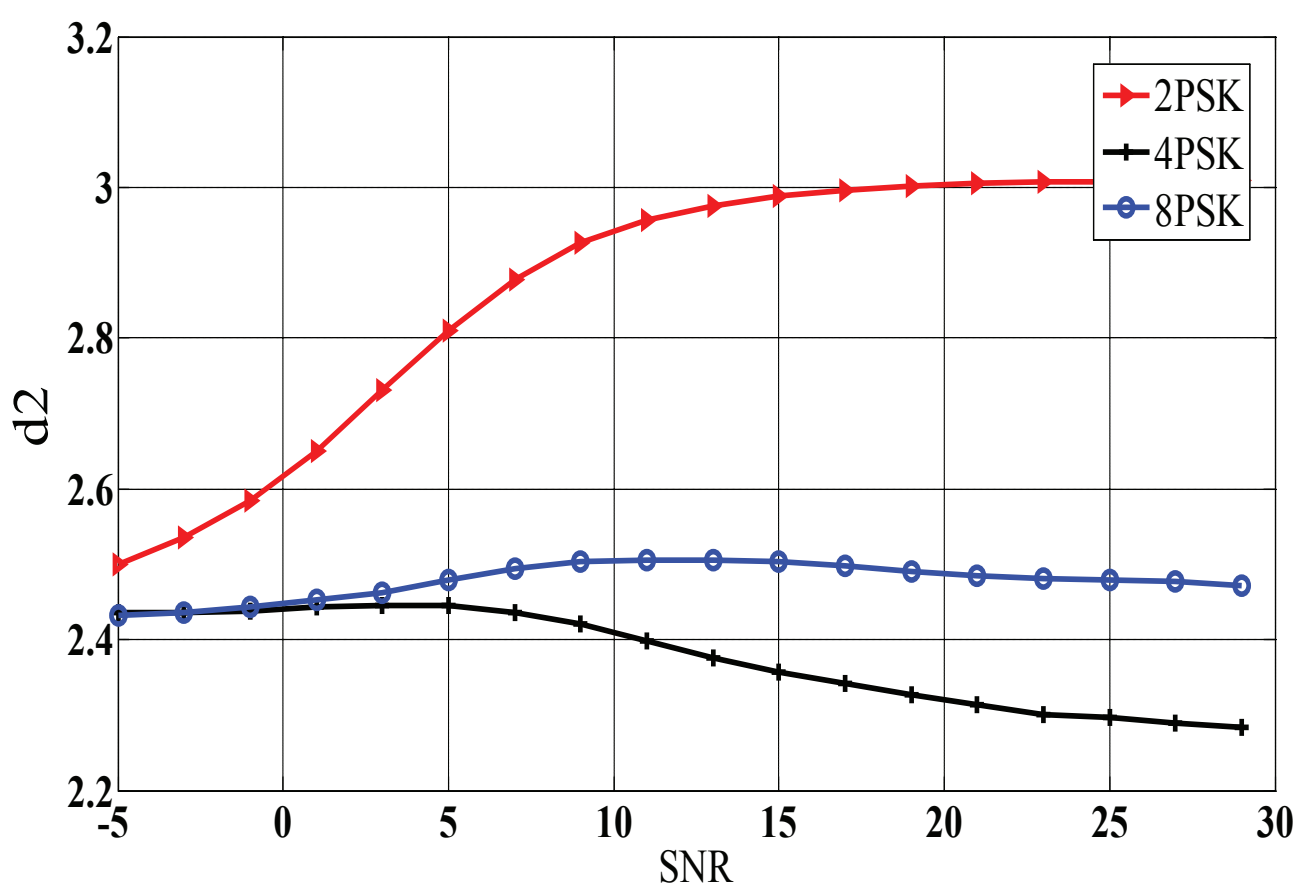

Figure $6 d_{2}$ for the discrimination between 2PSK and higher PSK signals. 


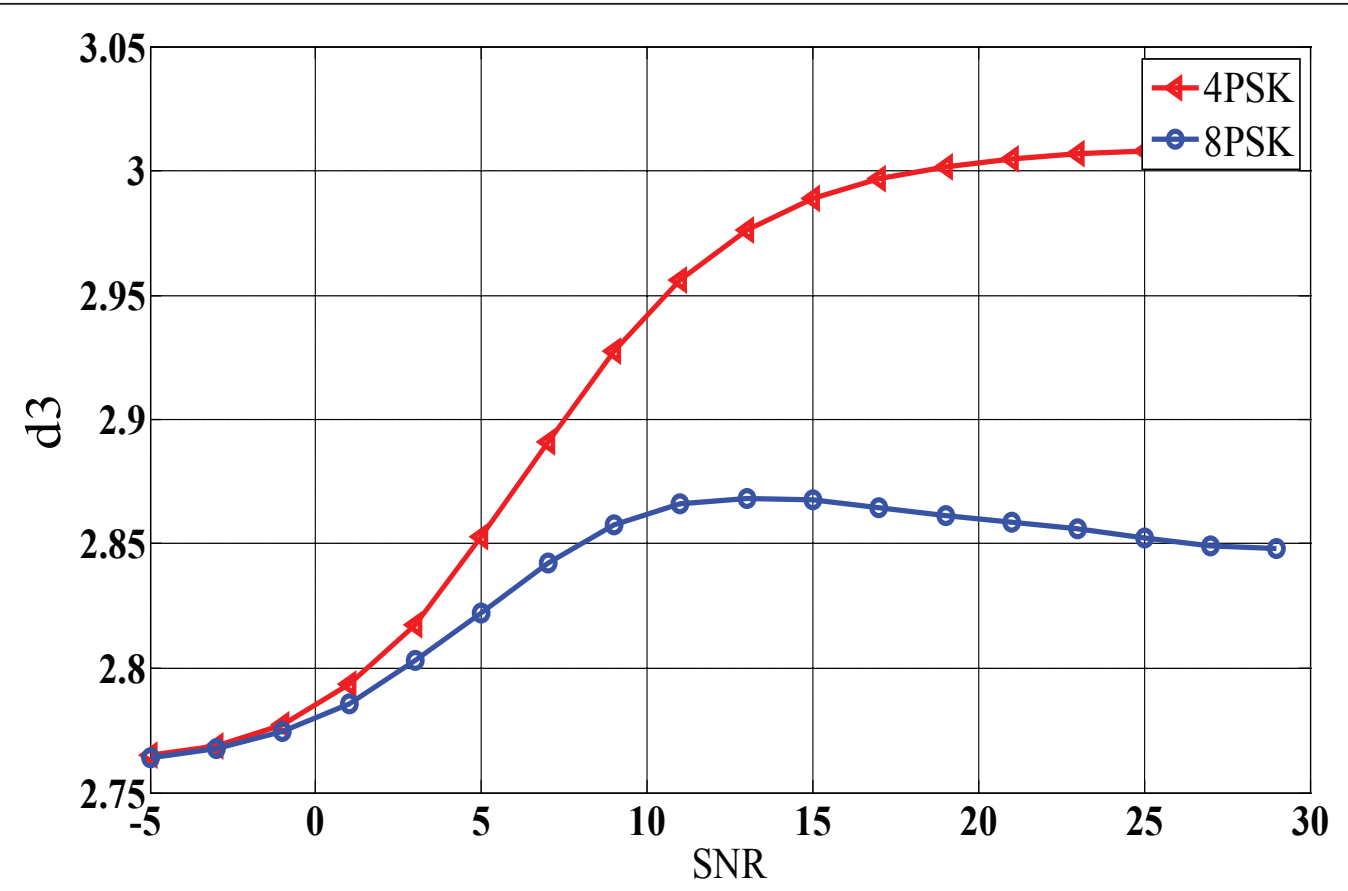

Figure $7 d_{3}$ for the discrimination between 4PSK and 8PSK signals.

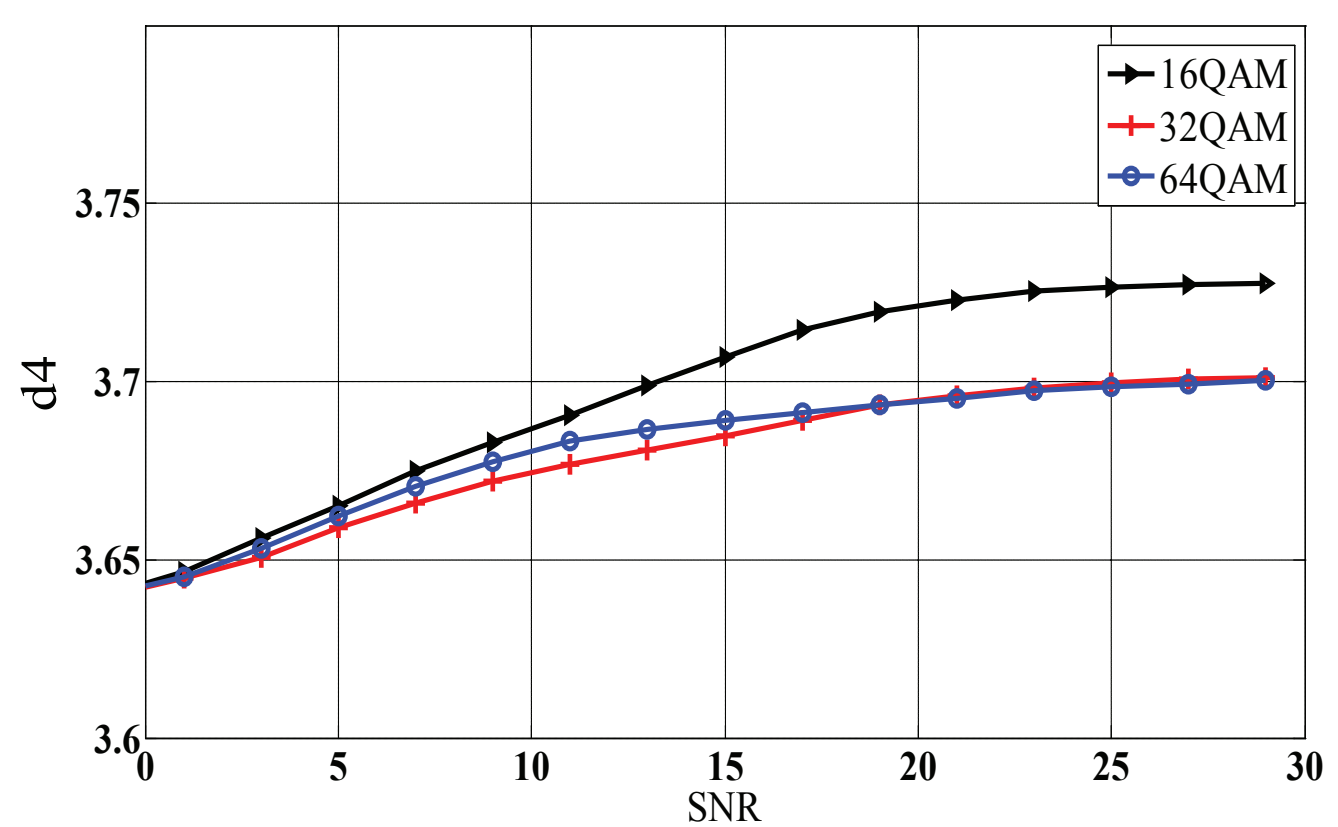

Figure $8 d_{4}$ for the discrimination between 16QAM and higher QAM signals. 


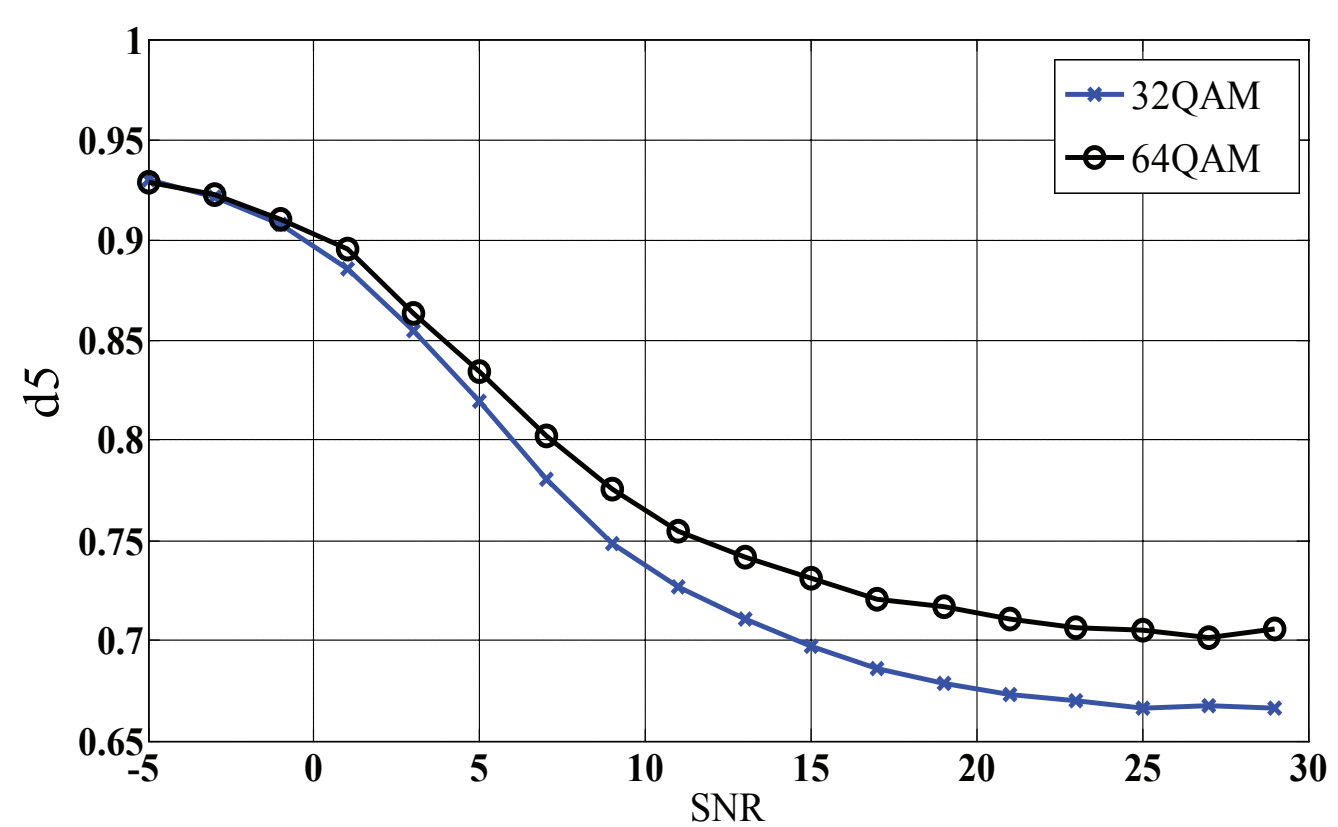

Figure $9 d_{5}$ for the discrimination between 32QAM and 64QAM

transmission [13]. SNR is adjusted by multiplying the output noise by the following factor:

$$
R_{s n r}=\sqrt{\frac{E}{N_{0}}} 10^{-S N R / 20}
$$

where $E, N_{0}$, SNR are the signal power, noise power, and desired SNR, respectively. All constellation points are normalized to zero mean and unity variance. The simulation parameters are given in Table 3.

For evaluation purposes, we measure the absolute value of the percentage deviation of each feature when noise model is changed from AWGN to bi-kappa. This percentage is evaluated using SNR ranging between 0 and $30 \mathrm{~dB}$, and is defined as follows

$$
\eta=\left|\frac{F_{\mathrm{AWGN}}-F_{\mathrm{Bi}-\text { kappa }}}{F_{\mathrm{AWGN}}}\right| \times 100
$$

where $F_{\mathrm{AWGN}}$ and $F_{\mathrm{Bi} \text {-kappa }}$ are the values of feature under consideration computed in the presence of AWGN and bi-kappa noise, respectively, at a particular

Table 3 Simulation parameters

\begin{tabular}{ll}
\hline Parameter & Value \\
\hline Carrier frequency & $f_{c}=24 \mathrm{kHz}$ \\
Symbol rate & $r_{s}=2400 \mathrm{~Hz}$ \\
Sampling rate & $f_{s}=153.6 \mathrm{kHz}$ \\
No. of symbols for testing & 512 \\
Total number of samples & 32768 \\
\hline
\end{tabular}

SNR. Figures 10, 11, 12, 13, 14, and 15 show the results, averaged over 100 independent realizations, for the following set of modulations: 2PSK, 4PSK, 8PSK, 16QAM, 32QAM, and 64QAM.

The above figures show that at SNR $<10 \mathrm{~dB}$, TTD and MDM are more robust than HOC against the change in HF noise model. It is true in general that $\eta$ decreases as SNR increases. However, for MPSK signals, the instantaneous amplitude feature has lower deviation for SNR $<30 \mathrm{~dB}$. This is intuitively not surprising because the difference between $F_{\mathrm{AWGN}}$ and $F_{\mathrm{bi} \text {-kappa }}$ relative to $F_{\mathrm{AWGN}}$ in this SNR range is smaller than that of higher SNR values. Another observation is that the wavelet based features have maintained almost the same values of $\eta$ for all considered modulations. In addition, the proposed MDM have shown excellent performance in the sense that they have the lowest deviation as compared to other features.

\section{Conclusions}

In this article, we have investigated the robustness of four features categories for the classification of digitally modulated signals in the presence of HF noise models; AWGN and bi-kappa noise. Specifically, the TTD, HOC, wavelets, and MDM features are considered, where the last feature is proposed in this work. It has been shown through computer simulations that $\mathrm{HOC}$ are sensitive to the change in noise model especially at low SNR $(<10$ $\mathrm{dB}$ ), while TTD, wavelets, and MDM show good robustness $(\eta<25 \%)$ in the investigated range of SNR. Note 


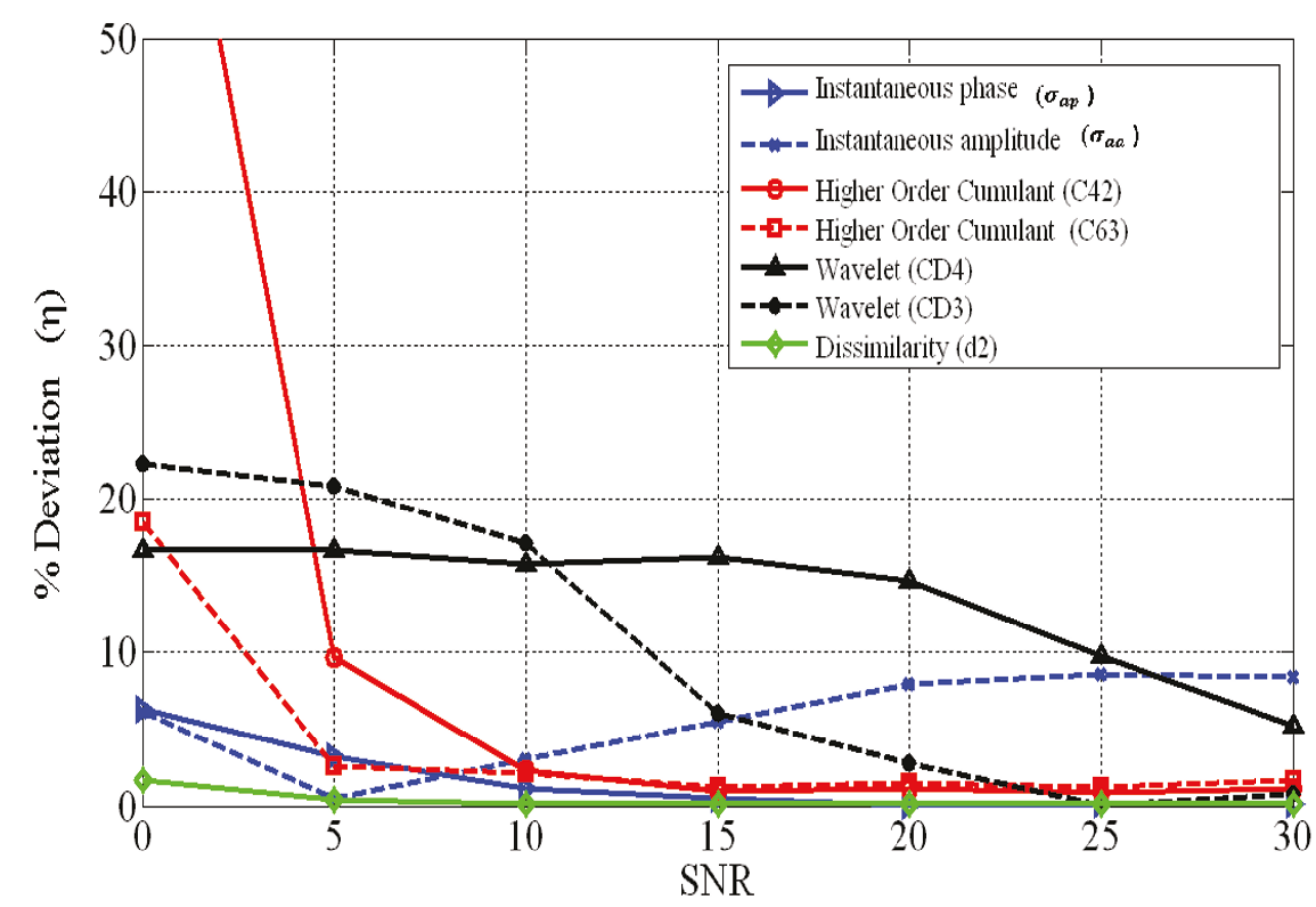

Figure 10 Features deviations computed from 2PSK signal.

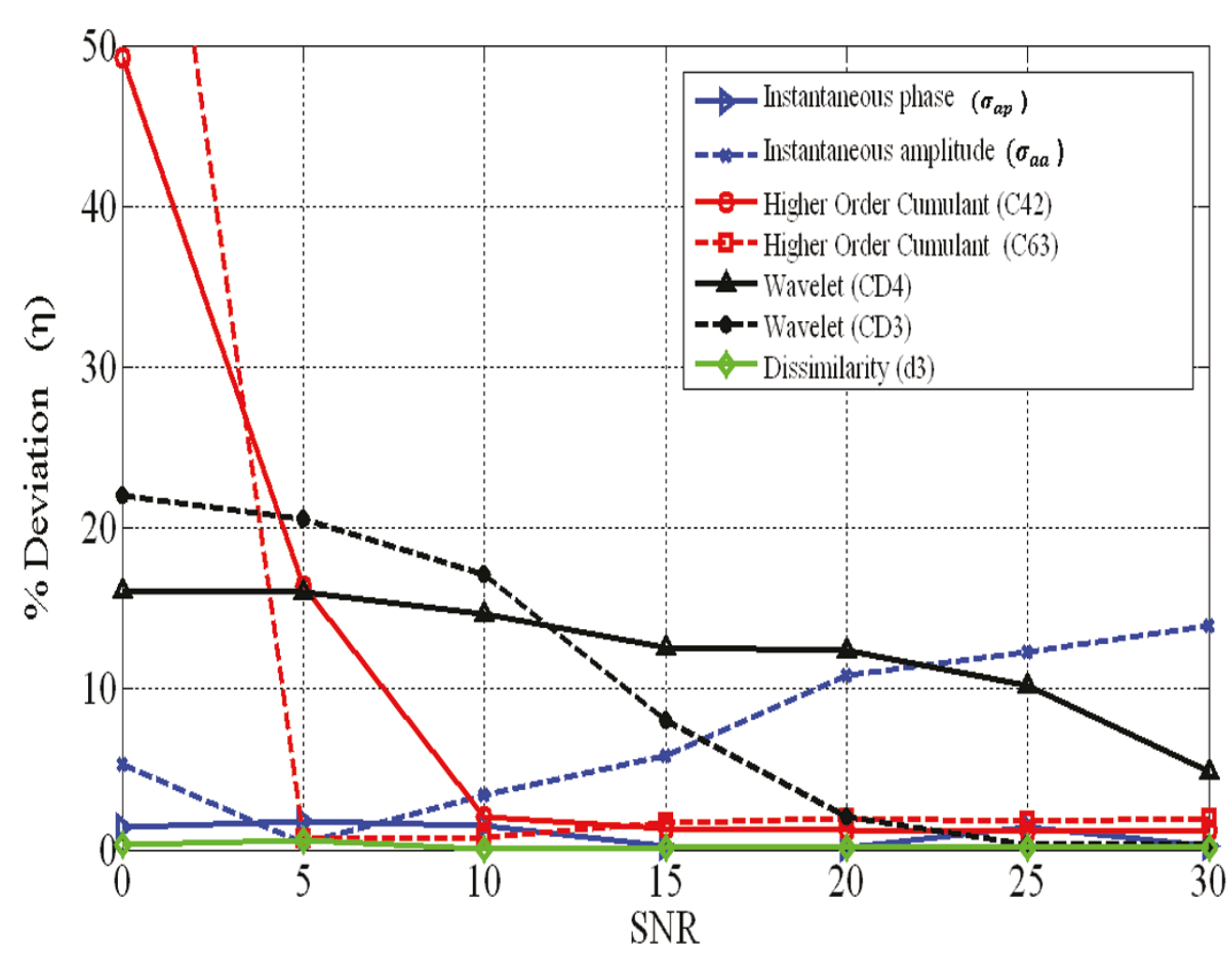

Figure 11 Features deviations computed from 4PSK signals. 


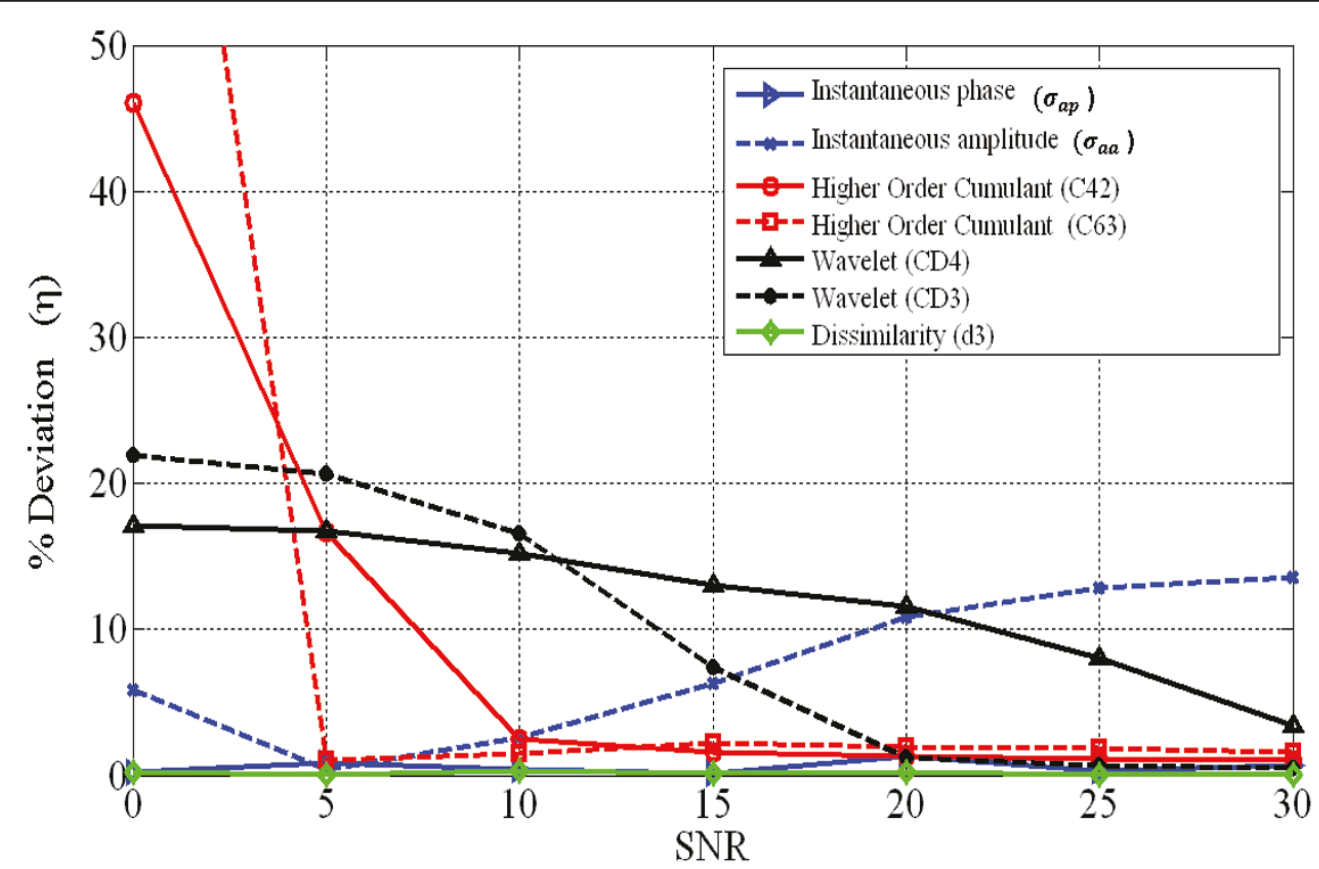

Figure 12 Features deviations computed from 8PSK signals.

that the proposed MDM features have the lowest values of $\eta$, i.e., highest robustness against variation in noise model, as compared to other features. The results of this article have potential values for the design of a classifier, as they identify the features that of higher robustness with respect to $\mathrm{HF}$ noise models. Note that the performance of an AMC designed under the assumption of AWGN noise model cannot be ensured when considering HF communications. Classifiers employing features sensitive to variation in noise model

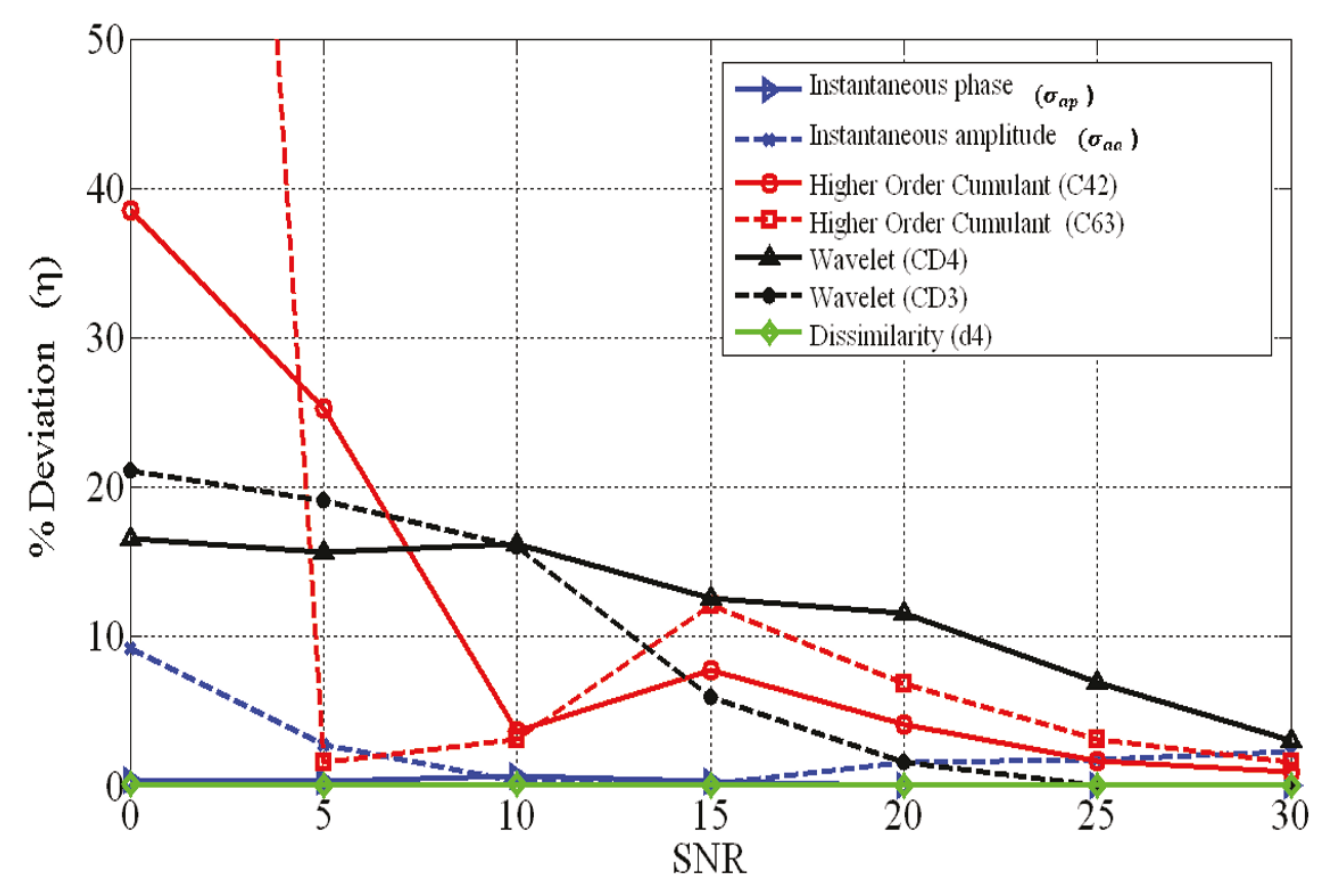

Figure 13 Features deviations computed from 16QAM signals. 


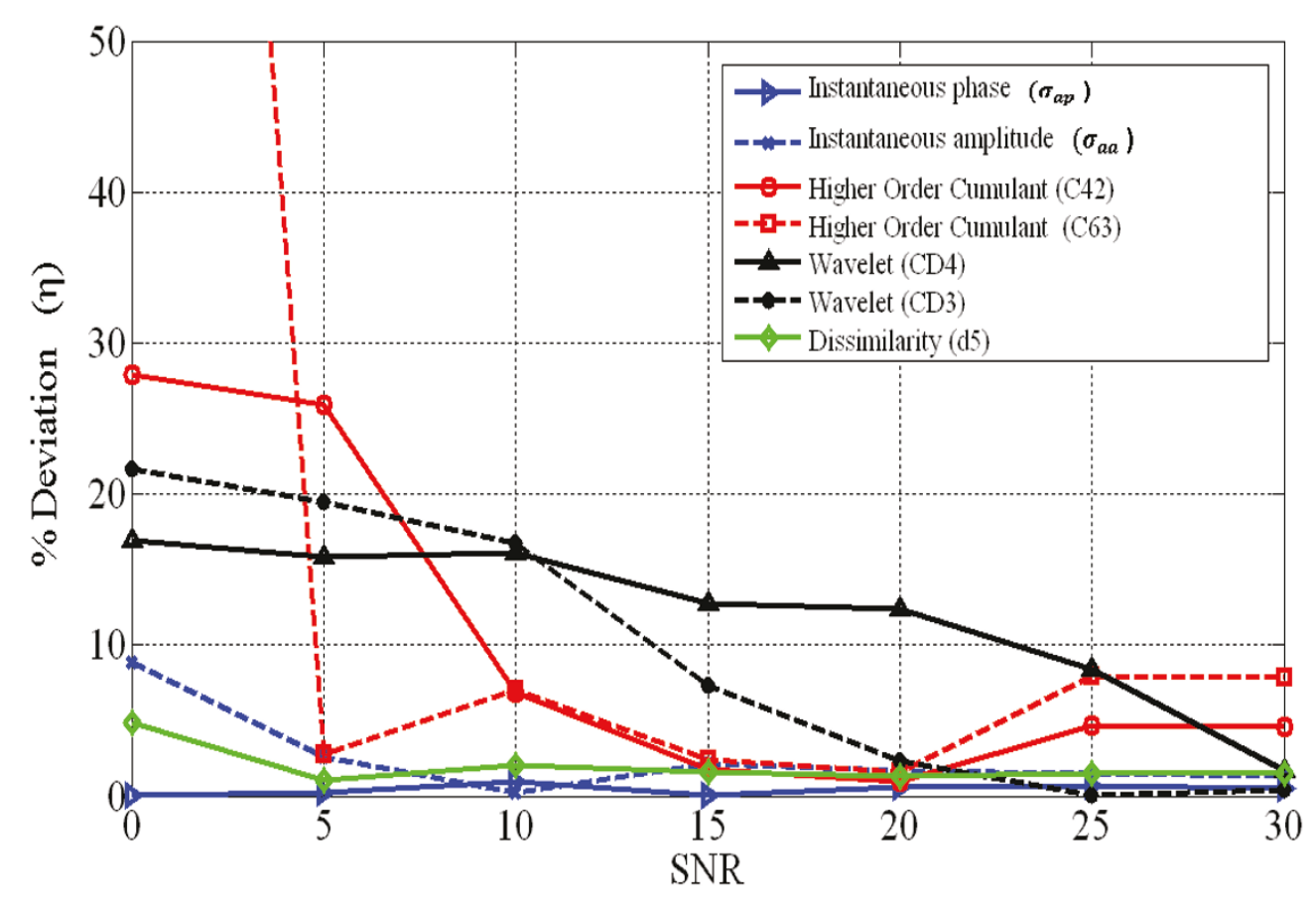

Figure 14 Features deviations computed from 32QAM signal.

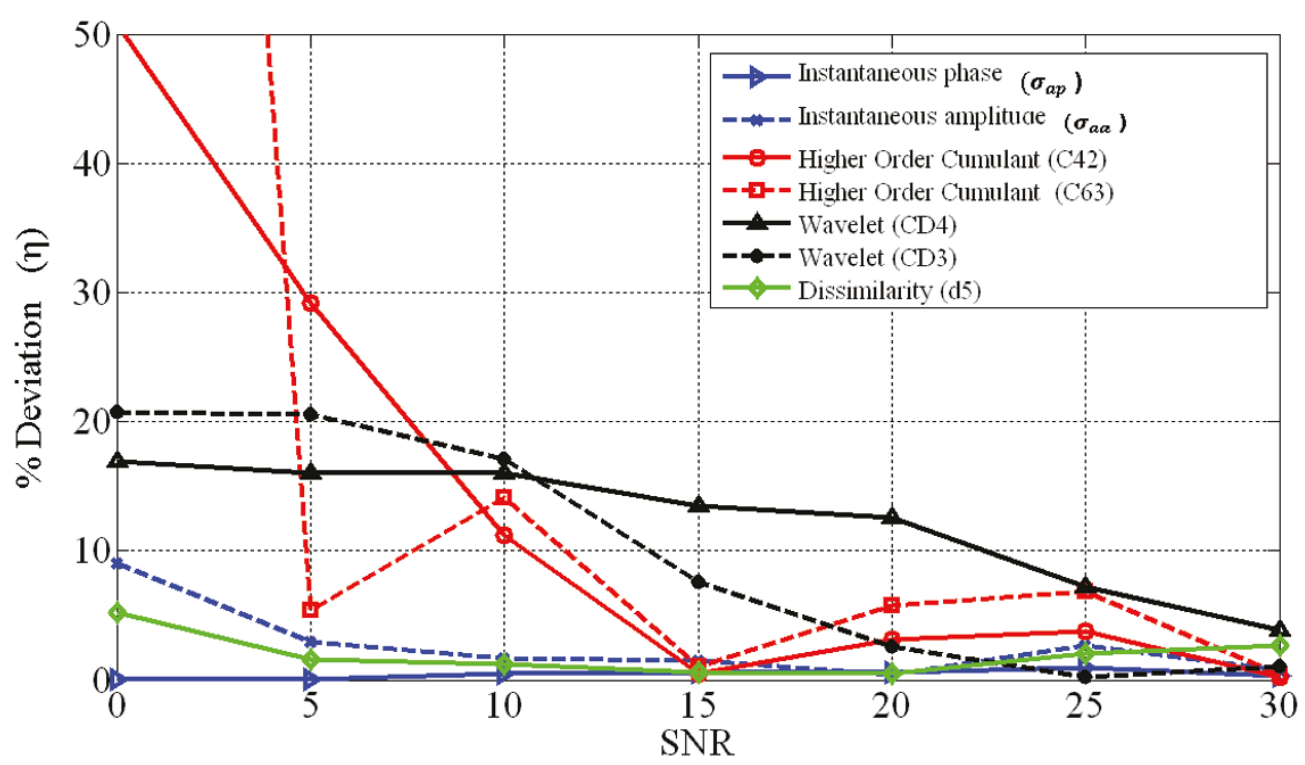

Figure 15 Features deviations computed from 64QAM modulation. 
should take this variation into consideration. For example, SVM or ANNs based classifiers need to be designed by training with signals corrupted by both HF noise models.

\section{List of Abbreviations}

AMC: Automatic modulation classification; ANNs: artificial neural networks; AWGN: additive white Gaussian noise; FB: feature based; HF: high frequency; HOC: higher order cumulants; LB: likelihood based; MDM: maximum dissimilarity measures; MLRT: maximum likelihood ratio test; PR: pattern recognition; SNR: signal-to-noise ratio; SVM: support vector machines; TTD: temporal time domain.

\section{Acknowledgements}

This work was supported by a grant (No. 08-ELE 263-2) from the unit of science and Technology at King Saud University.

\section{Author details}

'Electrical Engineering Department, College of Engineering, King Saud University, Riyadh, Saudi Arabia ${ }^{2}$ Prince Sultan Advanced Technologies Research Institute/STC-Chair, King Saud University, Riyadh, Saudi Arabia

\section{Competing interests}

The authors declare that they have no competing interests.

Received: 21 October 2010 Accepted: 29 June 2011

Published: 29 June 2011

\section{References}

1. P Jain, RM Buehrer, Implementation of adaptive modulation on the Sunrise software radio. in The Proceedings of the 45th Midwest Symposium on Circuits and Systems, 3, III-405-III-408, 4-7, August 2002

2. B Le, T Rondeau, D Maldonado, Modulation identification using neural networks for cognitive radios. in Proceedings of SDR05 Technical Conference, (Phoenix, AZ, 2005)

3. K Kim, IA Akbar, KK Bae, J Um, CM Spooner, JH Reed, Cyclostationary approaches to signal detection and classification in cognitive radio. in Proceedings of IEEE Dynamic Spectrum Access Nets. 212-215 (2007)

4. N Maslim, HF Communications: A Systems Approach. (CRC Press, London, 1987)

5. C Le Martret, D Boitea, Ageneral maximum likelihood classifier for modulation classification. in Proceedings of ICASSP, 4, 2165-2168 (1998)

6. OA Dobre, A Abdi, Y Bar-Ness, W Su, Survey of automatic modulation classification techniques: classical approaches and new trends. IET Commun. 1. 137-156 (2007)

7. MLD Wong, AK Nandi, Automatic digital modulation recognition using artificial neural network and genetic algorithm. Signal Process. 351-365 (2004)

8. P Cheol-Sun, J Won, N Sun-Phil, Automatic modulation recognition using support vector machine in software radio applications. in Proceedings of ICACT 2007, Gangwon-Do, 1, 9-12, February 2007

9. K Maliatsos, S Vassaki, Ph Constantinou, Interclass and intraclass modulation recognition using the wavelet transformation. in Proc PIMRC 2007, Athens, 1-5, September 2007

10. M Ling-Ling, S Xiu-jie, An improved algorithm of modulation classification for digital communication signals based on wavelet transform. in Proceedings of ICWAPR 07, Beijing, 3, 1226-1231, November 2007

11. Z Ye, G Memik, J Grosspietsch, Digital modulation classification using temporal waveform features for cognitive radios. in Proceedings of PIMRC 2007, Athens, 1-5, September 2007

12. AK Nandi, EE Azzouz, Algorithms for automatic modulation recognition of communication signals. IEEE Trans Commun. 46, 431-436 (1998)

13. EE Azzouz, AK Nandi, Automatic Modulation Recognition of Communication Signals, (Kluwer Academic, Norwell 1996)

14. T He, XR Jing, Modulation classification using ARBF networks. in Proceedings of ICSP, Beijing, China, 1809-18120, August 2004

15. L Peng, W Fuping, W Zanji, Algorithm for modulation recognition based on high-order cumulants and subspace decomposition. in Proceedings of 8th
International Conference on Signal Processing, Beijing, 3, 16-20, November 2006

16. OA Dobre, A Abdi, Y Bar-Ness, W Su, Selection combining for modulation recognition in fading channels. in Proceedings of IEEE MILCOM. Atlantic City, US, 2499-2505 (2005)

17. J Giesbrecht, R Clarke, D Abbott, An imperical study of the probability density function of HF noise. Fluct Noise Lett. 6(2), L117-L123 (2006)

18. J Giesbrecht, An empirical study of HF noise near Adelaide Australia. in IET 11th International Conference on Ionospheric Radio Systems and Techniques. Edinburgh, UK, 310-314, 28-30 April 2010

19. H Alharbi, S Mobien, S Alshebeili, F Alturki, Classification of digitally modulated signals in presence of non-Gaussian HF noise. in Proceedings of 7th ISWCS, Yourk, UK, September 2010, (in press)

20. R Prosch, Technical Handbook For Radio Monitoring I, (Books on Demand GmbH, Norderstedt, Germany, 2007)

21. JG Proakis, Digital Communications, (McGraw-Hill, New York, 2001)

22. P Cheol-Sun, C Jun-Ho, N Sun-Phil, J Won, Automatic modulation recognition of digital signals using wavelet features and SVM. in Proceedings of 10th ICACT. Gangwon-Do, 1, 387-390, February 2008

23. B Mobaseri, Constellation shape as a robust signature for digital modulation recognition. in Military Communications Conference Proceedings, MILCOM IEEE, 1, 442-446 (1999)

24. N Ahmadi, B Reza, Modulation classification of QAM and PSK from their constellation using Genetic Algorithm and hierarchical clustering. in 3d IEEE International Conference on Information \& Communication Technologies: from Theory to Applications, ICTTA'08. Umayyad Palace, Damascus, Syria, 7-11, April 2008

25. N Ahmadi, B Reza, A template matching approach to classification of QAM modulation using genetic algorithm. Signal Process Int J. 3(5), 95-109 (2009)

26. W Kam-Tim, K Chi-Wah, Clustering based distribution fitting algorithm for automatic modulation recognition. ISCC' 2007. pp. 13-18

27. Y Changyi, L Bingbing, L Yanling, Modulation classification of MQAM signals from their constellation using clustering. in 2010 Second International Conference on Communication Software and Networks, pp. 303-306

28. S Theodoridis, K Koutroumbas, Pattern Recognition, 4th edn. (Elsevier Inc Canada 2009)

doi:10.1186/1687-1499-2011-24

Cite this article as: Hazza et al:: Robustness of digitally modulated signal features against variation in HF noise model. EURASIP Journal on Wireless Communications and Networking 2011 2011:24

\section{Submit your manuscript to a SpringerOpen ${ }^{\mathcal{O}}$ journal and benefit from:}

- Convenient online submission

- Rigorous peer review

- Immediate publication on acceptance

- Open access: articles freely available online

- High visibility within the field

- Retaining the copyright to your article

Submit your next manuscript at $\gg$ springeropen.com 